\title{
Developing and Implementing Nursing Care Protocol Regarding Management of Postpartum Hemorrhage
}

\author{
Hind A. Sayed, Hamida A. Abd El hafez \& Ali M. El Saman. \\ 1. Nursing Specialist at Women's Health Hospital in Assiut University, Egypt. \\ 2. Assistant Professor of Obstetric \& Gynecologic Nursing, Faculty of Nursing, Assiut University, Egypt. \\ 3. Professor of Obstetrics \& Gynecological, Faculty of Medicin, Assiut University, Egypt .
}

\begin{abstract}
Background: Postpartum hemorrhage (PPH) is the leading cause of maternal morbidity and mortality in developing countries. PPH is defined as any blood loss $>500 \mathrm{ml}$ following vaginal delivery and $>1000 \mathrm{ml}$ after cesarean section. It can also be defined as fall in hematocrit $>10 \%$ or blood loss $>15 \%$ of total blood volume, or $10 \%$ measured per partum decline in hemoglobin levels. Aim of the study: the aim of this quasi-experimental study was to evaluate the effect of Developing and implementing Nursing Care protocol regarding management of postpartum hemorrhage on Patients outcomes and complications. Setting: This study was conducted at Assiut Women's Health Hospital, postpartum unit Sample: Purposive sample with a total of 140 Postpartum Hemorrhage patients was recruited, divided into two groups (control and study) 70 patients for each group Result: this study found that a significant difference between the routine and designed protocol in nursing management of postpartum hemorrhage . these findings show that the patient outcomes improved in study cases when compared with control cases which appeared in stop of bleeding $95.7 \%$ in study group, $82.8 \%$ in control group and conscious level $87.1 \%$ in study group,78.6 in control group with normal conscious level Conclusion: This research concluded that nursing care protocol was effective in reducing postpartum hemorrhage outcome and complications. Recommendations: clearly written Postpartum Hemorrhage management protocol, adapted for a particular hospital, should be developed and used in clinical practice.
\end{abstract}

\section{Keywords: Implementing, Nursing, Protocol, Management \& Postpartum Hemorrhage.}

\section{Introduction}

Globally, 14 million cases of postpartum hemorrhage occur annually (Rath, 2011). It is estimated that 18\% of all childbirth deliveries globally are affected by postpartum hemorrhage, and that severe hemorrhage affects $1-5 \%$ percent of all deliveries (Ganguli et al., 2011).

In Egypt, the maternal mortality ratio stood at 45deaths per 100, 000 live births in 2013 according to world health organization (WHO, 2014).

According to Ministry of Health \& population, September (2014) P.P.H. represents about $19.7 \%$ of the direct causes of maternal mortality, According to Egyptian Ministry of Health; maternal mortality rate is higher in Upper Egypt than Lower Egypt (74$61 / 100000$, respectively). Moreover, the most common cause of maternal mortality in Egypt is postpartum hemorrhage (19.7\%) (Kassebaum et al., 2014) Approximately $88 \%$ of these deaths occur within 4 hours of delivery, highlighting the need for timely management during delivery and in the immediate aftermath (Prata et al., 2014) Treatment delays and substandard care are often reported to be factors contributing to the high incidence of postpartum

hemorrhage and subsequent mortality (Woiski et al., 2015).
PPH is commonly defined as blood loss exceeding 1000 milliliters after vaginal birth or cesarean section. PPH is often classified as primary/immediate/early (occurring within 24 hours of birth) or secondary/delayed/late (occurring from more than 24 hours post birth to up to 12 weeks postpartum (American College of Obstetricians \& Gynecologists, 2016).

The most common cause is poor contraction of the uterus following childbirth. Not the entire placenta being delivered, a tear of the uterus, or poor blood clotting are other possible causes. It occurs more commonly in those who: already have a low amount of red blood, are Asian, with bigger or more than one baby, are obese or are older than 40 years of age. It also occurs more commonly following caesarean sections, those in whom medications are used to start labor, and those who have an episiotomy (Weeks, January 2015).

The usual presentation of PPH is one of heavy vaginal bleeding that can quickly lead to signs and symptoms of hypovolemic shock. This rapid blood loss reflects the combination of high uterine blood flow and the most common cause of PPH, i.e., uterine atony. Blood loss is usually visible at the introits, and this is especially true if the placenta has delivered. If the placenta remains in situ, then a significant amount of blood can be retained in the uterus behind a

Vol , (7) No , (17) June, 2019 
partially separated placenta, the membranes, or both (Schuurmans et al., 2015).

Physicians and nursing staff on labor and delivery should be skilled in accurately assessing blood loss and keenly aware that continued bleeding after delivery requires prompt evaluation and management. Quick recognition of early $\mathrm{PPH}$, along with identification of its cause and initiation of treatment, can avoid serious morbidity and mortality. Once PPH is diagnosed, the cause should be identified (Schneider et al, 2017).

Nurses are frequently the first responders to emergent events because they are readily at the patients' bedsides. It is necessary that they recognize the signs and symptoms of postpartum hemorrhage and that they know how to respond appropriately in these situations (Anonson et al., 2014)

The best patient outcome occurs when healthcare providers notice the subtle clinical changes in a patient that indicate the condition. Noticing these changes is the first step to better patient outcome; being able to implement the care required is the next step. When healthcare providers are able to quickly recognize these changes, patients are more likely to receive the appropriate care and more quickly recover from their condition. (Association of Women's Health, Obstetric \& Neonatal Nurses, 2013)

There is an increase in positive patient outcomes during treatment of postpartum hemorrhage after staff nurses attended training on the appropriate documentation and quantification of blood loss during the postpartum period (Ruth \& Kennedy, 2011).

Not surprisingly, many of the complications of severe $\mathrm{PPH}$ are related to massive blood loss and hypovolemic shock. Damage to all major organs is possible; respiratory (adult respiratory distress syndrome) and renal (acute tubular necrosis) damages are the most common but are rare. Pulmonary edema is uncommon in this previously healthy group; however, it may develop acutely or during the recovery phase because of fluid overload or myocardial dysfunction. (Wetta et al., 2013).

The WHO recommendations for the prevention of postpartum hemorrhage include the use of uterotonics during the third stage of labor. Uterotonics are drugs that result in adequate uterine contraction and can be used for prophylactic therapy or treatment (Munoz et al, 2012).

\section{Significance of the study}

Postpartum hemorrhage is a major cause of maternal death and morbidity in the United States and the world. (Berg, Callaghan, Syverson et al., 2010). In the United States, it complicates $1 \%$ to $5 \%$ of births (Francois \& Foley, 2017). Postpartum hemorrhage
$(\mathrm{PPH})$ is responsible for around $25 \%$ of maternal mortality worldwide, reaching as high as $60 \%$ in some countries (WHO, 2014).

Nurses are often the first line providers responding to postpartum hemorrhage because they are readily at the bedside. According to the Association of Women's Health, Obstetric and Neonatal Nurses (AWHONN), 93\% of maternal deaths caused by postpartum hemorrhage could have been prevented with improved clinical response (AWHONN, 2013). Assiut Governorate has the highest percentage of maternal mortality in Egypt (81 deaths/100 000 live births) ( Mahmoud, Omar, 2018).

\section{Aim of the study}

To evaluate the effect of developing and implementing Nursing care Protocol regarding management of postpartum hemorrhage on patient's outcomes and complications.

Research Hypothesis: Patients who receive nursing care protocol will improve Postpartum Hemorrhage Outcomes and complications.

Operational definition of postpartum hemorrhage outcomes: postpartum hemorrhage outcomes in this research were included the following; maternal recovery (bleeding and general condition improved), morbidity as renal failure, anemia

\section{Materials \& Methods}

Study design: A quasi-experimental design was used in the current study.

Setting: This study was conducted at postpartum words of obstetrics department in women's health center at Assiut university hospital which serves all cases rural and urban areas. This department is divided into Postpartum emergency sector which receives all women undergone C.S and normal delivery around the clock and Postpartum unit which divided into three units $(\mathrm{A}, \mathrm{B}, \mathrm{C})$ and include one nursing room for nine nurses and one supervisor and eight patient rooms which receive women after one hour of delivery till the discharge .

\section{Sample \& Subjects}

The study sample was selected by using the nonprobability sampling technique where a Purposive of $140 \mathrm{PPH}$ patient was recruited. All subjects should meet the specified inclusion criteria. These criteria include; Women with postpartum hemorrhage immediately post birth to 12 weeks, all modes of birth and all women accepted to participate in the study. The patients (140) divided to two groups: a control (routine care) and a study (nursing protocol) group. Each group70patients.

Exclusion criteria

1-Complicated postpartum hemorrhage patient.

2-Patient with blood diseases 


\section{Primary Outcome}

- Early management of postpartum hemorrhage.

\section{Secondary Outcomes}

- Preventing or reducing postpartum Hemorrhage complications.

\section{Tools of the study}

Two tools were designed and implemented by researcher after reviewing the related Journals, books and related publications and assessed for validity by a panel of 3 experts in the field (Jury) because there was not a clearly written Postpartum Hemorrhage nursing management protocol in the hospital and Modifications were carried out according to the expert's to collect the required data for this research:

Tool I: A Structured interviewing questionnaire it included questions related to personal data of participating patients which included name, age, address and obstetric history which included parity, gestational age, date of admission, mode of delivery, postpartum period, type of hemorrhage, cause of hemorrhage and date of discharge.

Tool II: Nursing care protocol in of PPH: this tool consists of three parts:-

\section{I. part (1)}

It included assessment of the patient vital signs, conscious level, amount of blood loss, fundal level

\section{Part (2):}

It included data related to nursing management of postpartum hemorrhage which included: ensure IV access, uterine massage, insert folly catheter, medication and blood product as ordered, keep the patient warm , attention to the patient response to resuscitation ,Monitor Complete blood count, coagulation studies and blood gases , assist to move to Operative room if indicated, documentation and reporting.

\section{Part (3)}

It included data related to post intervention assessment which included: general condition of the patient (vital signs, conscious level, bleeding).And presence of complications as (DIC , sepsis, transfusion or anesthetic reaction, fluid overload (pulmonary edema), anemia (normocytic normochromic, Sheehan syndrome).

Phases of the study: The current study proceeds systematically through the following phases:

\section{Administration phase}

The women were recruited from October 2016 to March 2017 after approving the study from ethical committee in the Faculty of Nursing and Medicine, Assiut University and obtaining an official permission from the council of department of obstetrics \&Gynecology in Woman's Health Hospital, Assiut University in order to obtain the acceptance and co-operation to be one of the team of management.

\section{Ethical consideration}

Oral consent was obtained from women who were participated in the study, after explaining the nature and purpose of the study. There was no any risk for the women during conduction of the study. The study was followed common ethical principles in clinical research. Confidentiality and anonymity would be assured and the participating women had the right to refuse participation or withdraw from the study without any rational.

\section{Tool Validity}

Validation of the tool was done through submission to the panel of 3 experts in the field (Jury). Modifications were carried out according to the expert's judgments.

\section{III.Pilot study}

A pilot study was carried out on $10 \%$ of the study sample (14 patients) to test the content clarity and applicability of the study tool and no modifications were done. Patients who included in the pilot study were also included in the current study.

\section{IV.Procedure}

Data were collected through a period from October 2016 till March 2017, each patient in the control and study group was individually interviewed to collect basic data using

Tool (1). Each patient assigned to one of the two groups as follows; the control group comprised 70 patients; were receiving the routine nursing care. The study group comprised 70 patients; were receiving nursing protocol. The control group will started with and completed before starting the study group. Nursing care and patients outcomes were evaluated for both control and study groups after intervention using Tool (2).

\section{Assessment of the patient}

The researcher started the assessment for patients immediately after admission; the patient assessed for general condition (conscious level) amount of blood loss either by a standardized absorbent sanitary pad (stained partly $(30 \mathrm{ml})$ ),

Saturated capacity $(100 \mathrm{ml}))$ OR by weighting materials $(1 \mathrm{gm}=1 \mathrm{ml})$, vital signs

(Especially pulse and BP), cause of bleeding (Tone, Trauma, Thrombin and tissue), fundal level and lochia, presence of complications.

\section{VI.Implementation phase}

This phase started immediately after assessment. The researcher with the team started Anti shock measures as, Ensure IV access to facilitate resuscitative measures. Insert Foley catheter to enhance bladder emptying and assess patient status and response, Monitor vital signs especially pulse, blood pressure to determine the degree of shock. Assess for tachypnea and tachycardia, Assess for signs of shock. Perform uterine massage in cases of 
atony to improve the uterine contractions. Mobilize additional staff as needed (Charge Nurse or Nurse Assistant ),Administer uterotonic medications as ordered ,Type and Cross - Consider use of Trauma blood if type and crossed blood not available ,Assign single person to communicate with blood bank. Maintain strict Intake \&Output, Weight materials, calculate and record cumulative blood loss q 5-15 minutes, Set up blood administration set and blood warmer for transfusion, Administer blood products and draw labs. Apply pulse oximeter / ECG monitoring, Elevate legs to 20-30 degree to improve the circulation to the vital organ, Keep patient warm, Anticipate and assess pain management need.

VII. Post Intervention follow up \&evaluation

The researcher continuously followed up the patients after implementation was carried out and the patient's condition was stabilized through Close attention to the response to resuscitation, Monitor Complete blood count, coagulation studies and blood gases. Assist with move the patient to Operative room (if indicated), the researcher finally documented and reporting all the assessment results.

\section{Statistical analysis}

Data were analyzed using statistical package for the social science (SPSS) version 20. Numerical data expressed as means and standard deviations. Qualitative data expressed as frequency and percentage. Chi-square test used to examine the relationship between qualitative variables. Repeated quantitative variables, unpaired $\mathrm{T}$ test used for comparison. Probability of error (p-value) $<0.05$ considered significant.

\section{Result}

Table (1): Comparison between the study group and control group according to personal characteristics.

\begin{tabular}{|c|c|c|c|c|c|}
\hline \multirow{3}{*}{ Age (years) } & \multicolumn{4}{|c|}{ Group } & \multirow{3}{*}{ P. value } \\
\hline & \multicolumn{2}{|c|}{ Study $(n=70)$} & \multicolumn{2}{|c|}{ Control $(n=70)$} & \\
\hline & No. & $\%$ & No. & $\%$ & \\
\hline$<25$ & 11 & 15.7 & 6 & 8.6 & \multirow{3}{*}{0.073} \\
\hline $25-30$ & 36 & 51.4 & 28 & 40.0 & \\
\hline$>\mathbf{3 0}$ & 23 & 32.9 & 36 & 51.4 & \\
\hline Mean \pm SD & \multicolumn{2}{|c|}{$29.59 \pm 5.32$} & \multicolumn{3}{|c|}{$31.06 \pm 5.63$} \\
\hline \multicolumn{6}{|l|}{ Residence } \\
\hline Rural & 19 & 27.1 & 22 & 31.4 & \multirow{2}{*}{0.577} \\
\hline Urban & 51 & 72.9 & 48 & 68.6 & \\
\hline
\end{tabular}

Table (2): Distribution of studied group according to their Obstetrical data.

\begin{tabular}{|c|c|c|c|c|c|}
\hline & \multicolumn{2}{|c|}{ Study $(n=70)$} & \multicolumn{2}{|c|}{ Control $(n=70)$} & \multirow{2}{*}{ P-value } \\
\hline & No. & $\%$ & No. & $\%$ & \\
\hline \multicolumn{5}{|l|}{ Parity: } & \multirow{3}{*}{0.086} \\
\hline $1-3$ & 46 & 65.7 & 36 & 51.4 & \\
\hline$<3$ & 24 & 34.3 & 34 & 48.6 & \\
\hline \multicolumn{5}{|c|}{ Gestational age: (weeks) } & \multirow{4}{*}{0.475} \\
\hline$<36$ & 15 & 21.4 & 10 & 14.3 & \\
\hline $36-37$ & 32 & 45.7 & 32 & 45.7 & \\
\hline 38 or more & 23 & 32.9 & 28 & 40.0 & \\
\hline \multicolumn{5}{|c|}{ Mode of delivery: } & \multirow{3}{*}{0.858} \\
\hline NVD & 23 & 32.9 & 24 & 34.3 & \\
\hline $\mathrm{CS}$ & 47 & 67.1 & 46 & 65.7 & \\
\hline \multicolumn{5}{|c|}{ Type of PPH: } & \multirow{3}{*}{1.000} \\
\hline Primary & 67 & 95.7 & 67 & 95.7 & \\
\hline Secondary & 3 & 4.3 & 3 & 4.3 & \\
\hline \multicolumn{6}{|c|}{ Cause of PPH: } \\
\hline Atonic & 57 & 81.4 & 50 & 71.4 & 0.163 \\
\hline Traumatic & 12 & 17.1 & 20 & 28.6 & 0.107 \\
\hline Infection & 1 & 1.4 & 0 & 0.0 & 1.000 \\
\hline
\end{tabular}

NVD: Normal vaginal delivery

CS: Cesearian section

Vol , (7) No, (17) June, 2019 
Table (3): Comparison between study group and control group according to Nursing management of postpartum hemorrhage (Assessment part).

\begin{tabular}{|c|c|c|c|c|c|c|c|c|c|}
\hline \multirow{3}{*}{ Variables } & \multicolumn{4}{|c|}{$\operatorname{Study}(n=70)$} & \multicolumn{4}{|c|}{ Control(n=70) } & \multirow{3}{*}{ P-value } \\
\hline & \multicolumn{2}{|c|}{ Done } & \multicolumn{2}{|c|}{ Not done } & \multicolumn{2}{|c|}{ Done } & \multicolumn{2}{|c|}{ Not done } & \\
\hline & No. & $\%$ & No. & $\%$ & No. & $\%$ & No. & $\%$ & \\
\hline \multicolumn{10}{|l|}{ Assess blood loss which include } \\
\hline Amount & 70 & 100.0 & 0 & 0.0 & 30 & 42.9 & 40 & 57.1 & $0.000^{*}$ \\
\hline Source & 70 & 100.0 & 0 & 0.0 & 43 & 61.4 & 27 & 38.6 & $0.000 *$ \\
\hline Cause & 70 & 100.0 & 0 & 0.0 & 20 & 28.6 & 50 & 71.4 & $0.000 *$ \\
\hline \multicolumn{10}{|l|}{ Monitor vital signs which included } \\
\hline Pulse & 70 & 100.0 & 0 & 0.0 & 52 & 74.3 & 18 & 25.7 & $0.000^{*}$ \\
\hline $\mathrm{BP}$ & 70 & 100.0 & 0 & 0.0 & 38 & 54.3 & 32 & 45.7 & $0.000 *$ \\
\hline RR & 70 & 100.0 & 0 & 0.0 & 22 & 31.4 & 48 & 68.6 & $0.000^{*}$ \\
\hline Temperature & 70 & 100.0 & 0 & 0.0 & 15 & 21.4 & 55 & 78.6 & $0.000^{*}$ \\
\hline Ensure IV access & 70 & 100.0 & 0 & 0.0 & 70 & 100.0 & 0 & 0.0 & -- \\
\hline $\begin{array}{l}\text { Assess for tachypnea and } \\
\text { tachycardia }\end{array}$ & 70 & 100.0 & 0 & 0.0 & 37 & 52.9 & 33 & 47.1 & $0.000 *$ \\
\hline $\begin{array}{l}\text { Assess for narrowed pulse } \\
\text { pressure, hypotension and cool } \\
\text { extremities }\end{array}$ & 70 & 100.0 & 0 & 0.0 & 48 & 68.6 & 22 & 31.4 & $0.000 *$ \\
\hline $\begin{array}{l}\text { Assess location and consistency of } \\
\text { the fundus }\end{array}$ & 70 & 100.0 & 0 & 0.0 & 33 & 47.1 & 37 & 52.9 & $0.000 *$ \\
\hline
\end{tabular}

*: statistically significant $\quad$-IV: intravenous $\quad-R R$ : Respiratory Rate $\quad$-Bp: Blood pressure

Table (4): Comparison between study group and control group according To nursing management of postpartum hemorrhage (Intervention part).

\begin{tabular}{|c|c|c|c|c|c|c|c|c|c|}
\hline & \multicolumn{4}{|c|}{$\operatorname{Study}(n=70)$} & \multicolumn{4}{|c|}{ Control $(n=70)$} & \multirow{3}{*}{ P-value } \\
\hline & \multicolumn{2}{|c|}{ Done } & \multicolumn{2}{|c|}{ Not done } & \multicolumn{2}{|c|}{ Done } & \multicolumn{2}{|c|}{ Not done } & \\
\hline & No. & $\%$ & No. & $\%$ & No. & $\%$ & No. & $\%$ & \\
\hline $\begin{array}{l}\text { Insert fully catheter and } \\
\text { empty the bladder }\end{array}$ & 70 & 100.0 & 0 & 0.0 & 46 & 65.7 & 24 & 34.3 & $0.000^{*}$ \\
\hline Perform uterine massage & 70 & 100.0 & 0 & 0.0 & 44 & 62.9 & 26 & 37.1 & $0.000^{*}$ \\
\hline Mobilize additional staff as needed & 70 & 100.0 & 0 & 0.0 & 27 & 38.6 & 43 & 61.4 & $0.000^{*}$ \\
\hline $\begin{array}{l}\text { Administer uterotonic medications } \\
\text { a s ordered }\end{array}$ & 70 & 100.0 & 0 & 0.0 & 55 & 78.6 & 15 & 21.4 & $0.000^{*}$ \\
\hline $\begin{array}{l}\text { Type and cross-consider use of } \\
\text { general blood bank if type and } \\
\text { Crossed blood not available. }\end{array}$ & 70 & 100.0 & 0 & 0.0 & 14 & 20.0 & 56 & 80.0 & $0.000^{*}$ \\
\hline $\begin{array}{l}\text { Assign single person to } \\
\text { communicate with blood bank }\end{array}$ & 70 & 100.0 & 0 & 0.0 & 26 & 37.1 & 44 & 62.9 & $0.000^{*}$ \\
\hline Maintain strict I\&O & 70 & 100.0 & 0 & 0.0 & 28 & 40.0 & 42 & 60.0 & $0.000^{*}$ \\
\hline $\begin{array}{l}\text { Weigh materials, calculate and } \\
\text { record cumulative blood } \\
\text { loss q 5-15 minutes }\end{array}$ & 67 & 95.7 & 3 & 4.3 & 5 & 7.1 & 65 & 92.9 & $0.000^{*}$ \\
\hline $\begin{array}{l}\text { Use fluid warmer and/or rapid } \\
\text { infuser for fluid \& blood product } \\
\text { administration }\end{array}$ & 65 & 92.9 & 5 & 7.1 & 12 & 17.1 & 58 & 82.9 & $0.000^{*}$ \\
\hline $\begin{array}{l}\text { Apply pulse oximeter/ ECG } \\
\text { monitoring }\end{array}$ & 70 & 100.0 & 0 & 0.0 & 62 & 88.6 & 8 & 11.4 & $0.006^{*}$ \\
\hline Elevate legs to 20-30 degree & 61 & 87.1 & 9 & 12.9 & 15 & 21.4 & 55 & 78.6 & $0.000^{*}$ \\
\hline Keep the patient warm & 70 & 100.0 & 0 & 0.0 & 19 & 27.1 & 51 & 72.9 & $0.000^{*}$ \\
\hline $\begin{array}{l}\text { Anticipate and assess pain } \\
\text { management need }\end{array}$ & 70 & 100.0 & 0 & 0.0 & 12 & 17.1 & 58 & 82.9 & $0.000 *$ \\
\hline
\end{tabular}

*: statistically significant 
Table (5): Distribution of Patients according to outcomes in both groups.

\begin{tabular}{|c|c|c|c|c|c|}
\hline & \multicolumn{2}{|c|}{ Study $(n=70)$} & \multicolumn{2}{|c|}{ Control $(n=70)$} & \multirow{2}{*}{ P-value } \\
\hline & No. & $\%$ & No. & $\%$ & \\
\hline \multicolumn{5}{|l|}{ Bleeding: } & \multirow{3}{*}{$0.003^{*}$} \\
\hline Stopped & 67 & 95.7 & 58 & 82.8 & \\
\hline Not stopped & 3 & 4.3 & 12 & 17.2 & \\
\hline \multicolumn{5}{|c|}{ Conscious level: } & \multirow{3}{*}{$0.001 *$} \\
\hline Comatose & 9 & 12.9 & 15 & 21.4 & \\
\hline Conscious & 61 & 87.1 & 55 & 78.6 & \\
\hline
\end{tabular}

*: statistically significant

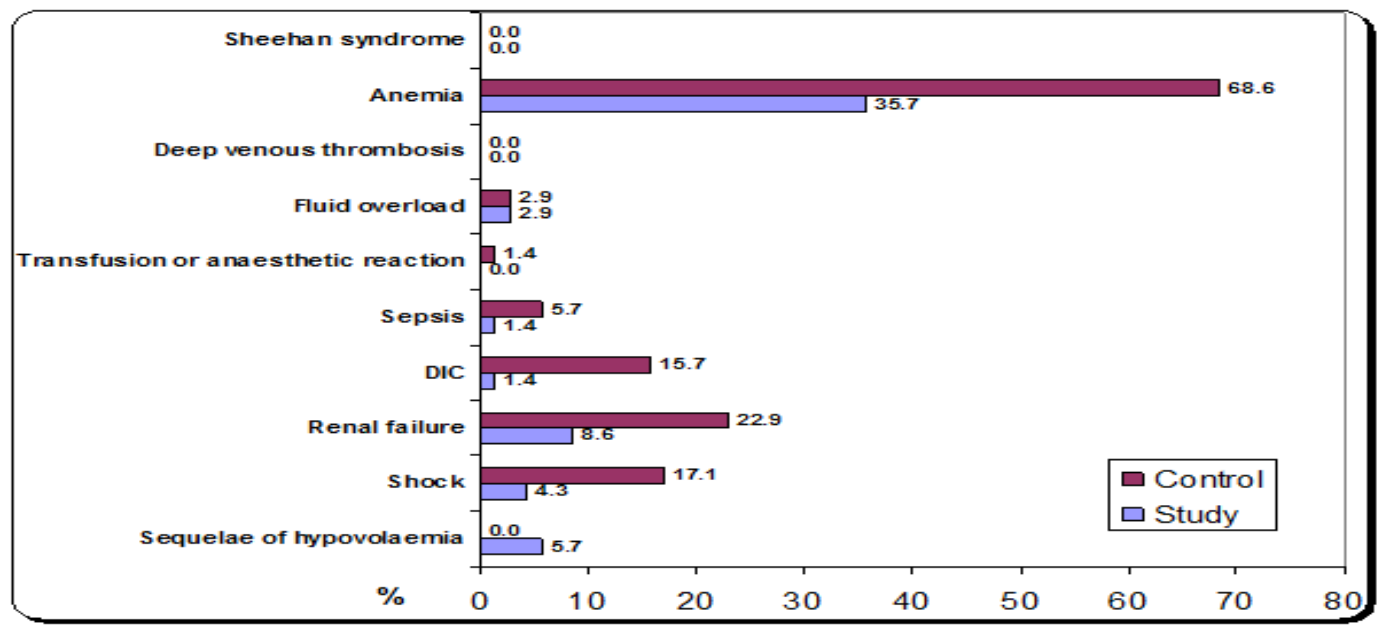

Figure (1):Distribution of patients in Both groups according to complications.

Table (1): Comparing the personal characteristics between study and control group in which there was no statistical difference as regard age, residence.

Table (2): Show that the most common cause of PPH was uterine atony $(76.42 \%)$, the most common type of $\mathrm{PPH}$ is primary $\mathrm{ppH}(95 \%)$ and $(66.4 \%)$ of women with PPH were delivered by emergency CS compared with only $(33.6 \%)$ delivered by NVD.

Table (3): Shows the comparison in the nursing intervention of PPH cases in study and control group which reflected that there is a highly statistically significant Difference between planned nursing management and routine nursing management in cases of PPH.

Table(4): Show the comparison in the nursing assessment of PPH cases in study and control group which reflected that there is a highly statistically significant Difference between designed nursing assessment and routine nursing assessment in cases of PPH

Table(5): Show that the patient outcomes improved in study cases when compared with control cases which appeared in stop of bleeding( $95.7 \%$ )in study group, $(82.8 \%)$ in control group and conscious level(87.1\%)in study group,(78.6)in control group with normal conscious level.
Figure (1): Show decreased patients complications in study group when compared with study group, shock (4.3\% in study group and $17.1 \%$ in control group), Renal failure $(8.6 \%$ in study group and $22.9 \%$ in control group), DIC (1.4\% in study group and $15.7 \%$ in control group), Anemia (35.7\% in study and $68.6 \%$ in control group).

\section{Discussion}

In this research, researcher attempted to find the effect of nursing care protocol on postpartum hemorrhage patient's outcomes. The findings of this research supported the research hypothesis which is "Patients who receive nursing care protocol will improve Postpartum Hemorrhage Outcomes".

(Farquhar et al., \& Geller et al., 2014) identified contributory and avoidable factors of maternal deaths, including organizational and staff factors such as inadequate education and training, or lack of staff knowledge. Two other studies have reported less than optimal management of severe PPH and failure to apply guidelines fully in approximately $40 \%$ of cases, partly due to maternity unit status. These studies used retrospective medical records, however, and it is difficult to control for case mix in retrospective chart 
reviews. Furthermore, chart abstraction underestimates the quality of care.

The findings of this research indicated that there was a statistically significant difference between two groups regard initial assessment ( amount of blood loss , source of bleeding, cause of bleeding, Blood pressure, location and consistency of the fundus), the assessment of the patients was an important point in early management and improving the outcomes, this results are supported by American Journal of Maternal \& Child Nursing (2013) who concluded that the accurate assessment of postpartum hemorrhage patient and blood loss facilitates timely transfusion and reduce severity of hemorrhagic shock, and this also is consistent with Miller study(2014), who concluded that the best patient outcomes occur when guidelines, resources and education are provided to healthcare providers. Equipping bedside nurses with the knowledge and resources needed to make appropriate decision during emergent situation will lead to better patient outcomes.

Findings of this research indicated that there was a statistically significant difference between two groups as regard performing uterine massage, weight materials, calculate and record cumulative blood loss, maintain strict intake \&output which could affect on the prognosis of the patients and their outcomes.

Current result of this study indicated that the percentage of weighting materials as accurate method in calculating amount of blood loss in control group was less than $10 \%$ which can delaying in proper management. These findings are in accordance with the studies carried out by Gabel \& Weeber (2012) found that staff nurses continuously underestimate blood loss when assessing a patient's Lochia (bleeding) after delivery. This underestimation of blood loss leads to improper management of postpartum hemorrhage and in (Al Kadri et al., 2011) reported the use of weighting of blood loss collected on a sensitive scale, to diagnose immediate PPH is a crucial step for early management and improved outcomes. Which also agreed by (Roston, 2012) Experts have suggested that improving the accuracy and reliability of blood loss estimation is the 'crucial step' in early diagnosis of PPH and that most deaths from PPH could be avoided through 'appropriate diagnosis'.

Findings of this research indicated that there was a statistically significance difference between study and control group in stopping of bleeding which was more than half in study group, less than in control group This is consistent with several previous studies, where the decrease in the PPH and severe $\mathrm{PPH}$ rates was reported after the implementation of the new PPH management guideline.
Current results of this study indicate that the patient complications decreased following the implementation of protocol when compared with control group as regard anemia over more than half of patients in control and more than one third in study group, Disseminated intravascular coagulopathy was over $15 \%$ in control and below $2 \%$ in study group, renal failure was over $20 \%$ in control and below $10 \%$ in study group. this results are in agree with (Roston et al., 2012) who denoted that Delays in the treatment of PPH are believed to have a direct effect on the severity of bleeding, the development of complications such as coagulopathy and increasing rates of morbidity and mortality. Delays are reported to be caused by misinterpretation of the extent of blood loss and its physiological effects, failure to recognize hidden bleeding, and failure to escalate care to more senior colleagues.

\section{Conclusion}

Based on result of the present study; it could be concluded that:

- The proposed nursing protocol for postpartum Hemorrhage patients was effective in reducing patient's outcomes and complications.

- This study also revealed that both the guidelines and the way of implementation have led to a better diagnosis and treatment of PPH. It also has led to a more conservative approach to the treatment of postpartum hemorrhage that has less negative effect on quality of life.

\section{Recommendations}

1. Based on the result of the present study, the researcher suggested the following recommendations:

Postpartum hemorrhage $(\mathrm{PPH})$ remains one of the most common causes of maternal morbidity and mortality. Therefore, clearly written $\mathrm{PPH}$ management guidelines should be used in clinical practice.

2. all nurses in obstetrics and gynecology departments especially those at postpartum department should be trained in managing postpartum hemorrhage as a team member.

3. Hospitals should develop postpartum hemorrhage projects designed to increase awareness associated of postpartum hemorrhage mortality and morbidity by improving nurses' recognition of, readiness for, and response to a postpartum hemorrhage.

\section{References}

1. A COG Practice Bulletin (2016): Clinical Management Guidelines for Obstetrician- 
Gynecologists

hemorrhage.108:1039-47

2. Al Kadri H., Al Anazi B., Tamim H., (2011): Visual estimation versus gravimetric measurement of postpartum blood loss: vol (283):pp1207-13

3. American Journal of Maternal and Child Nursing (2013).

4. Anonson, J., Walker, M., (2014): Qualities of exemplary nurse leaders. Journal of Nursing Management, 22(1), 127-136

5. Association of Women's Health, Obstetric and Neonatal Nurses. (2013)

6. Cohain, J., Kappa (2012): A Novel Way to Prevent Postpartum Hemorrhage. Midwifery Matters, 135, 21-22.

7. Farquhar C., Sadler L., Masson V., (2011): Classifying contributory factors and potentially avoidable maternal deaths in New Zealand, 2006-2009: 331(8). 1-8.

8. Francois K., Foley M., (2017): Antepartum and Postpartum

9. Hemorrhage. Chapter 18. pp. 395-424.

10. Gabel, K., \& Weeber, T., (2012): Measuring and communicating blood loss during obstetric hemorrhage. Journal of Obstetric, Gynecologic \& Neonatal Nursing, 41(4), 551-558.

11. Ganguli, S., Stecker M., Pyne D., Baum R., Fan C., (2011): Uterine Artery Embolization In The Treatment Of Postpartum Uterine Hemorrhage. Journal of Vascular and Interventional Radiology, 22(2), 169-176.

12. Geller S., Koch A., Martin N., Rosenberg D., (2014): Assessing preventability of maternal mortality in Illinois: 2002-2012. 211 (698).e111.

13. Hogan M., Foreman K., Naghavi M., (2010): Maternal mortality for 181 countries, vol (375):pp1609-1623.

14. Kassebaum N., Amelia B., Megan S., Katya A., Caitlyn S., Kyle R., (2014): Global, regional, and national levels and causes of maternal mortality during 1990-2013: a systematic analysis for the Global Burden of Disease study , 384: 980-1004.

15. Leach, L., \& Mayo, A., (2013): Rapid response teams: Qualitative

16. Analysis of their effectiveness, 22 (3), 198-210.

17. Mahmoud G., Omar A., (2018): Women's awareness and perceptions about maternal mortality in rural communities in Assiut and Fayoum Governorates, Egypt. 15(1)21-9.

18. Miller, K., (2014): The effect of simulation activities on maternal-newborn knowledge in a practical nursing course: Implications for practice, 29 (1), 41-45.
19. Munoz, Luis Cabero-Roura (2012): Problems in Determining Accurate Rates of Postpartum Hemorrhage, 34(8), 5.

20. Rath W., (2011): Postpartum hemorrhage update on problems of definitions and diagnosis. (90), 421-8.

21. Roston A., Roston A., Patel A., (2012): Blood loss: Accuracy of Visual Estimation. pp71-72

22. Ruth, D., \& Kennedy, B., (2011): Acute volume resuscitation following obstetric hemorrhage, 25 (3), 253-260.

23. Prata N., Sreenivas A., Vahidnia F., Potts M., (2014): Saving maternal lives in resource-poor settings: Facing reality ;89 (2) :131-148

24. Schneider, K., \& Belfort, M., (2017): Postpartum Hemorrhage, vol (46), pp543-547.

25. Weeks, A., (January 2015): "The prevention and treatment of postpartum haemorrhage, 122 (2):202-10

26. Wetta A., \& Jeff M., (2013): Risk Factors for Uterine Atony \Postpartum Hemorrhage Requiring Treatment after Vaginal Delivery 209(1):51

27. WHO (2012): Recommendations for the prevention and treatment of PPH, Geneva, Switzerland, pp: 1-48.

28. WHO (2014): Guidelines for management of PPH and retained placenta.

29. WHO (2014): trends in maternal Mortality: 1990 to 2013

30. Woiski M., Scheepers H., Liefers J., (2015): Guideline-based development of quality indicators for prevention and management of postpartum hemorrhage. ; 94(10):1118-1127. 